\title{
Research Article \\ Spatiality of Derivations of Operator Algebras in Banach Spaces
}

\author{
Quanyuan Chen ${ }^{1,2}$ and Xiaochun Fang ${ }^{1}$ \\ ${ }^{1}$ Department of Mathematics, Tongji University, Shanghai 200092, China \\ ${ }^{2}$ Department of Information Engineering, Jingdezhen Ceramic Institute, Jingdezhen, Jiangxi 333403, China
}

Correspondence should be addressed to Quanyuan Chen, cqy0798@163.com

Received 5 June 2011; Revised 17 August 2011; Accepted 9 September 2011

Academic Editor: Wolfgang Ruess

Copyright (c) 2011 Q. Chen and X. Fang. This is an open access article distributed under the Creative Commons Attribution License, which permits unrestricted use, distribution, and reproduction in any medium, provided the original work is properly cited.

Suppose that $\mathcal{A}$ is a transitive subalgebra of $B(X)$ and its norm closure $\bar{A}$ contains a nonzero minimal left ideal $\supset$. It is shown that if $\delta$ is a bounded reflexive transitive derivation from $\mathcal{A}$ into $B(X)$, then $\delta$ is spatial and implemented uniquely; that is, there exists $T \in B(X)$ such that $\delta(A)=T A-A T$ for each $A \in \mathcal{A}$, and the implementation $T$ of $\delta$ is unique only up to an additive constant. This extends a result of E. Kissin that "if $\bar{A}$ contains the ideal $C(H)$ of all compact operators in $B(H)$, then a bounded reflexive transitive derivation from $A$ into $B(H)$ is spatial and implemented uniquely." in an algebraic direction and provides an alternative proof of it. It is also shown that a bounded reflexive transitive derivation from $\mathcal{A}$ into $B(X)$ is spatial and implemented uniquely, if $X$ is a reflexive Banach space and $\bar{A}$ contains a nonzero minimal right ideal $\supset$.

\section{Introduction}

Throughout this paper, $X$ is a Banach space (and $X$ will be replaced by $H$ if it is a Hilbert space) and $\mathcal{A}$ is a subalgebra of $B(X)$, the Banach algebra of all bounded operators on $X$. Suppose that $\mathcal{A} \subseteq B(X)$ and $\mathcal{M} \subseteq B(X)$ is an $\mathcal{A}$-bimodule. A linear map $\delta$ from $\mathcal{A}$ into $\mathcal{M}$ is called a derivation if

$$
\delta(A B)=\delta(A) B+A \delta(B) \text { for any } A, B \in \mathcal{A} .
$$

Then $A$ is called the domain of $\delta$ and denoted by $\operatorname{Dom}(\delta)$. The derivation $\delta$ is called inner (resp., spatial) if there exists an operator $T \in \mathcal{M}$ (resp., $T \in B(X)$ ) such that

$$
\delta(A)=T A-A T \quad \text { for any } A \in \mathcal{A} .
$$


If the operator $T$ is not bounded, then $\delta$ is said to be quasispatial. More precisely, if there exists a densely defined, closed operator $T: \operatorname{Dom}(T) \rightarrow X$ such that

$$
A(\operatorname{Dom}(T)) \subseteq \operatorname{Dom}(T), \quad \delta(A) x=(T A-A T) x \quad \text { for any } A \in \mathcal{A}, x \in \operatorname{Dom}(T),
$$

then the derivation $\delta$ is called quasispatial, and the operator $T$ is an implementation of $\delta$. Compared to the spatiality, the quasispatiality is a slightly weaker notion.

Given a bounded derivation $\delta$ on an operator algebra, the natural question is whether the derivation $\delta$ is inner (or spatial). The spatiality of derivations is a classical problem when formulated for self-adjoint algebras and non-self-adjoint reflexive operator algebras. And it has been extensively studied in the literature in a large variety of situations, and some interesting results have been obtained [1-13]. For example, every derivation of a $C^{*}$-algebra is spatial [12], every derivation of a von Neumann algebra is inner [13], and so is the derivation of a nest algebra [14]. Every derivation from an atom Boolean subspace lattice algebra into its ideal is quasispatial [7]. A necessary and sufficient condition is given for a derivation on CDC algebras to be quasispatial [6]. In [10], the quasispatiality of derivations on CSL algebras is studied.

As to general operator algebras, it is well known that every derivation of $B(X)$ is inner [9] and that every derivation of a standard operator subalgebra on a normed space $X$ is spatial [4]. Since these operator algebras are transitive, the above question for a reflexive transitive derivation in a Banach space $X$ is raised naturally as follows.

Problem 1. Suppose that $\mathcal{A}$ is a transitive subalgebra of $B(X)$. Let $\delta$ be a bounded reflexive transitive derivation from $\mathcal{A}$ into $B(X)$. Does there always exist $T \in B(X)$ such that $\delta(A)=T A-A T$ for each $A \in \mathcal{A}$ ? Is the implementation $T$ unique only up to an additive constant if there is any?

In the case when $X$ is a Hilbert space, it is Problem 2.12 of [5]. Although the problem is still open, some strong conditions have been found by Kissin to imply that such derivations are spatial and implemented uniquely (Proposition 2.11, [5]). In particular, Kissin has proved that the answer to Problem 1 is affirmative under the conditions that $\mathcal{A}$ is a transitive subalgebra of $B(H)$, and $\bar{A}$ contains the ideal $C(H)$ of all compact operators in $B(H)$. As far as we know, there are no other solution to Problem 1.

The purpose of this paper is to investigate the quasispatiality of derivations and to address the above question in a Banach space $X$. The paper is organized as follows. In Section 2, we give some preliminaries. In Section 3, we investigate the quasispatiality and spatiality of derivations. The main result (Theorem 3.1) shows that if $\mathscr{A}$ is a transitive operator algebra on a Banach space $X$ and $\bar{A}$ contains a nonzero minimal left ideal $\supset$, then a bounded reflexive transitive derivation $\delta$ from $\mathcal{A}$ into $B(X)$ is spatial and implemented uniquely. As an application, the quasispatiality of the adjoint of a derivation is discussed in Section 4. The main result (Theorem 4.2) in Section 4 shows that under the conditions that $X$ is a reflexive Banach space and $\bar{A}$ contains a nonzero minimal right ideal $\supset, \delta$ is also spatial and implemented uniquely if $\delta$ is a bounded reflexive transitive derivation from $A$ into $B(X)$. As another application, Proposition 2.11 (ii) of [5] can be proved by using Theorem 3.1 of this paper, which is Corollary 4.3. Since Theorem 2.5 and Proposition 2.11 of [5] hold in a Hilbert space and they are not valid in a Banach space without the approximation property, Theorems 3.1 and 4.2 of this paper extend the result of Kissin to Banach spaces in an algebraic direction. 
The role of "compact operators" is replaced by that of "minimal one-sided ideals". The proof of our results relies on the quasispatiality of the derivation and Banach algebra techniques.

This paper is a continuation of [5]. Some definitions and notations can be found in [5].

\section{Preliminaries}

Throughout this paper, $X$ is a complex Banach space, and $X^{*}$ is the topological dual space of $X$, the Banach space of all continuous linear functionals on $X$. We denote by $\mathcal{F}(X)$ the algebra of all finite-rank operators on $X$. If a subalgebra $\mathcal{A}$ contains $\mathcal{F}(X)$, then $\mathcal{A}$ is called a standard operator algebra. For a bounded operator $A$ on $X$, denote by Lat $A$ the lattice of all closed invariant subspaces of $A$ and $A^{*}$ the adjoint operator of $A$. For a subalgebra $A$ of $B(X)$, denote by Lat $(\mathcal{A})$ the lattice of all closed subspaces invariant under every operator in $\mathcal{A}$. For a set $\mathcal{L}$ of subspaces of $X$, denote by $\operatorname{Alg} \mathcal{L}$ the algebra of all operators in $B(X)$ which leave all subspaces in $\mathcal{L}$ invariant. An operator algebra $\mathcal{A}$ is transitive if Lat $\mathcal{A}=\{\{0\}, X\} ; \mathcal{A}$ is reflexive if

$$
\mathcal{A}=\text { Alg Lat } \mathcal{A}, \quad \text { where Alg Lat } \mathcal{A}=\{T \in B(X): \text { Lat } \mathcal{A} \subset \operatorname{Lat} T\} .
$$

For $0 \neq x \in X$ and $0 \neq f \in X^{*}$, the rank-one operator $x \otimes f$ acts on $X$ by $(x \otimes f) y=f(y) x$ for $y \in X$. Let $A$ be an operator on $X$ with $\operatorname{Dom}(A) \subseteq X$. If $x \in \operatorname{Dom}(A)$ and $f \in \operatorname{Dom}\left(A^{*}\right)$, then $A(x \otimes f)=A x \otimes f$ and $(x \otimes f) A=x \otimes\left(A^{*} f\right)$. Let $M$ be a nonempty subset of $X$ and $N$ a nonempty subset of $X^{*}$. The annihilator $M^{\perp}$ of $M$ and the preannihilator ${ }^{\perp} N$ of $N$ are defined as follows [15]: $M^{\perp}=\left\{f \in X^{*}: f(x)=0\right.$ for all $\left.x \in M\right\},{ }^{\perp} N=\{x \in X: f(x)=0$ for all $f \in$ $N$ \}. It is obvious that $M^{\perp}$ is a weak*-closed subspace of $X^{*}$ and ${ }^{\perp} N$ is a norm-closed subspace of $X$. For a subalgebra $A$ and a closed, densely defined operator $T$ with domain $\operatorname{Dom}(T)$, we say that Tcommutes with $\mathcal{A}$, if $A(\operatorname{Dom}(T)) \subseteq \operatorname{Dom}(T)$ and $T A \xi=A T \xi$ for any $A \in \mathcal{A}$ and any $\xi \in \operatorname{Dom}(T)$. For a subalgebra $\mathcal{A}$ of $B(X)$, let $\mathscr{A}^{*}=\left\{A^{*}: A \in \mathcal{A}\right\}$ in notation.

A subset $\supset$ of an algebra $A$ is a left ideal of $\mathcal{A}$ if $A \supset \subseteq \supset$, a right ideal if $\supset \mathcal{A} \subseteq \supset$, and a two-sided ideal if it is both a left and a right ideal. A left ideal $\supset$ of $\mathscr{A}$ is minimal if every left ideal of $\mathcal{A}$ included in $\supset$ is either $\supset$ or $\{0\}$, similarly for minimal right ideals.

A derivation $\delta$ is bounded (resp., closed) if the map $\operatorname{Dom}(\delta) \ni A \mapsto \delta(A) \in B(X)$ is bounded (resp., closed) in the operator norm topology. The derivation $\delta$ is transitive if its domain $\operatorname{Dom}(\delta)$ is a transitive operator algebra; $\delta$ is reflexive if

$$
\mathcal{A}_{\delta}=\left\{\widehat{A}=\left(\begin{array}{cc}
A & \delta(A) \\
0 & A
\end{array}\right): A \in \operatorname{Dom}(\delta)\right\}
$$

is a reflexive operator algebra on $X \oplus X$. Denote by $\operatorname{Imp}(\delta)$ the set of all closed, densely defined operators implementing the derivation $\delta$ as in (1.3). For a densely defined, closed operator $T$ with domain $\operatorname{Dom}(T)$, we can define the derivation $\Delta_{T}$ with domain

$$
\operatorname{Dom}\left(\Delta_{T}\right)=\{A \in B(X): A(\operatorname{Dom}(T)) \subseteq \operatorname{Dom}(T), T A-A T \text { is bounded on } \operatorname{Dom}(T)\}
$$


by

$$
\Delta_{T}(A)=\operatorname{Closure}(T A-A T), \quad \text { for any } A \in \operatorname{Dom}\left(\Delta_{T}\right) .
$$

If a densely defined, closed operator $T$ implements a derivation $\delta$, then the derivation $\Delta_{T}$ is an extension of the derivation $\delta$. In fact, $A \in \operatorname{Dom}(\delta)$ implies $A \in \operatorname{Dom}\left(\Delta_{T}\right)$ and $\Delta_{T}(A)=\delta(A)$ for any $A \in \operatorname{Dom}(\delta)$ by (1.3) and (2.4). For any set $\Lambda$ of derivations $\delta_{\lambda}(\lambda \in \Lambda)$, define the derivation $\delta_{\Lambda}=\bigcap_{\lambda \in \Lambda} \delta_{\lambda}$ by

$$
\begin{gathered}
\operatorname{Dom}\left(\delta_{\Lambda}\right)=\left\{A \in \bigcap_{\lambda \in \Lambda} \operatorname{Dom}\left(\delta_{\lambda}\right): \delta_{\lambda}(A) \text { coincide for any } \lambda \in \Lambda\right\} \\
\delta_{\Lambda}(A)=\delta_{\lambda}(A) \text { for any } A \in \operatorname{Dom}\left(\delta_{\Lambda}\right) \text { and any } \lambda \in \Lambda .
\end{gathered}
$$

In particular,

$$
\delta_{\operatorname{Imp}(\delta)}=\bigcap_{T \in \operatorname{Imp}(\delta)} \Delta_{T}
$$

Remark 2.1. $\Delta_{T}$ is a reflexive transitive derivation for any densely defined, closed operator $T$. Indeed, $\operatorname{Dom}\left(\Delta_{T}\right)$ is a subalgebra of $B(X)$, and $\Delta_{T}$ is a derivation.

If $x \in \operatorname{Dom}(T)$ and $h \in \operatorname{Dom}\left(T^{*}\right),(x \otimes h) z=h(z) x \in \operatorname{Dom}(T)$ for any $z \in \operatorname{Dom}(T)$. So $(x \otimes h)(\operatorname{Dom}(T)) \subseteq \operatorname{Dom}(T)$. Since $(T(x \otimes h)-(x \otimes h) T)(z)=(T x \otimes h) z-\left(x \otimes T^{*} h\right) z$, $\|T(x \otimes h)-(x \otimes h) T\| \leq\|T x\|\|h\|+\|x\|\left\|T^{*} h\right\|$ so that $T(x \otimes h)-(x \otimes h) T$ is bounded on $\operatorname{Dom}(T)$. It follows that $x \otimes h \in \operatorname{Dom}\left(\Delta_{T}\right)$ for any $x \in \operatorname{Dom}(T)$ and $h \in \operatorname{Dom}\left(T^{*}\right)$.

Let $M \in \operatorname{Lat}\left(\operatorname{Dom}\left(\Delta_{T}\right)\right)$ with $0 \neq x_{0} \in M$. Then $(x \otimes h)\left(x_{0}\right)=h\left(x_{0}\right) x \in M$ for any $x \in \operatorname{Dom}(T)$ and $h \in \operatorname{Dom}\left(T^{*}\right)$. Since $\operatorname{Dom}\left(T^{*}\right)$ is dense, there exists $h_{0} \in \operatorname{Dom}\left(T^{*}\right)$ such that $h_{0}\left(x_{0}\right) \neq 0$ by Hahn-Banach Theorem. It follows that $x \in M$ for any $x \in \operatorname{Dom}(T)$ so that $\operatorname{Dom}(T) \subseteq M$. Thus $X=\overline{\operatorname{Dom}(T)} \subseteq \bar{M}=M$. Therefore $\operatorname{Dom}\left(\Delta_{T}\right)$ is transitive.

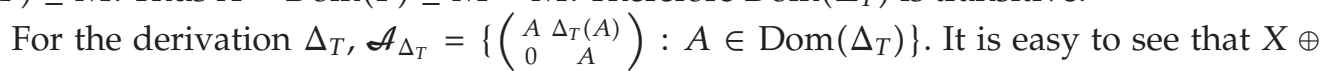
$\{0\} \in \operatorname{Lat}\left(\mathcal{A}_{\Delta_{T}}\right)$ and $G(T+t I)=\{(T(x)+t x): x \in \operatorname{Dom}(T)\} \in \operatorname{Lat}\left(\mathcal{A}_{\Delta_{T}}\right)$ for any $t \in \mathbb{C}$. Let $B_{2}=\left(\begin{array}{ll}B_{11} & B_{12} \\ B_{21} & B_{22}\end{array}\right) \in B(X \oplus X)$ and $B_{2} \in \operatorname{Alg} \operatorname{Lat}\left(\mathcal{A}_{\Delta_{T}}\right)$. Then $\operatorname{Lat}\left(\mathcal{A}_{\Delta_{T}}\right) \subseteq \operatorname{Lat}\left(B_{2}\right)$. Since $X \oplus\{0\} \in$ $\operatorname{Lat}\left(\mathcal{A}_{\Delta_{T}}\right) \subseteq \operatorname{Lat}\left(B_{2}\right),\left(\begin{array}{ll}B_{11} & B_{12} \\ B_{21} & B_{22}\end{array}\right)\left(\begin{array}{l}x \\ 0\end{array}\right)=\left(\begin{array}{l}B_{11} x \\ B_{21} x\end{array}\right) \in X \oplus\{0\}$ for any $x \in X$. It follows that $B_{21} X=\{0\}$. Thus $B_{21}=0$ and $B_{2}=\left(\begin{array}{cc}B_{11} & B_{12} \\ 0 & B_{22}\end{array}\right)$. Since $G(T+t I) \in \operatorname{Lat}\left(\mathcal{A}_{\Delta_{T}}\right) \subseteq \operatorname{Lat}\left(B_{2}\right),\left(\begin{array}{cc}B_{11} & B_{12} \\ 0 & B_{22}\end{array}\right)\left(\begin{array}{c}T(x)+t x \\ x\end{array}\right)=$ $\left(\begin{array}{c}B_{11} T x+t B_{11} x+B_{12} x \\ B_{22} x\end{array}\right) \in G(T+t I)$ for any $x \in \operatorname{Dom}(T)$ and any $t \in \mathbb{C}$. Then $B_{22} x \in \operatorname{Dom}(T)$ and $B_{11} T x+t B_{11} x+B_{12} x=(T+t I) B_{22} x$ for any $x \in \operatorname{Dom}(T)$ and any $t \in \mathbb{C}$. It follows that $B_{11} x=$ $B_{22} x \in \operatorname{Dom}(T)$ and $B_{11} T x+B_{12} x=T B_{22} x$ for all $x \in \operatorname{Dom}(T)$. Since $\operatorname{Dom}(T)$ is dense in $X$ and $B_{11}, B_{22} \in B(X), B_{11}=B_{22}$. So $B_{11}(\operatorname{Dom}(T)) \subseteq \operatorname{Dom}(T)$ and $B_{12}=T B_{11}-B_{11} T$ on $\operatorname{Dom}(T)$. Therefore, $B_{12}=T B_{11}-B_{11} T$ is bounded on $\operatorname{Dom}(T)$. It follows that $B_{11}=B_{22} \in \operatorname{Dom}\left(\Delta_{T}\right)$ and $B_{12}=\Delta_{T}\left(B_{11}\right)$. Thus $B_{2}=\left(\begin{array}{cc}B_{11} & \Delta_{T}\left(B_{11}\right) \\ 0 & B_{11}\end{array}\right) \in \mathcal{A}_{\Delta_{T}}$. It follows that $\mathcal{A}_{\Delta_{T}}$ is a reflexive algebra. Therefore $\Delta_{T}$ is a reflexive transitive derivation. 


\section{Main Results}

In this section, we discuss the quasispatiality of bounded transitive derivations on operator algebras in a Banach space. The main result is as follows.

Theorem 3.1. Suppose that $\mathcal{A}$ is a transitive subalgebra of $B(X)$ and $\bar{A}$, the norm closure of $\mathcal{A}$, contains a nonzero minimal left ideal $\supset$. If $\delta$ is a bounded reflexive transitive derivation from $A$ into $B(X)$, then $\delta$ is spatial and implemented uniquely, or, more precisely, there exists $T \in B(X)$ such that $\delta(A)=T A-A T$ for each $A \in \mathcal{A}$ and the implementation $T$ is unique only up to an additive constant.

The proof of Theorem 3.1 will proceed through several lemmas, in each of which we maintain the same notation. The results similar to the following two lemmas (Lemmas 3.2 and 3.3) can be found in [5]. For the sake of completeness, we outline the proof.

Lemma 3.2. Let $\delta$ be a reflexive transitive derivation from $\operatorname{Dom}(\delta)(\operatorname{Dom}(\delta)=\mathscr{A} \subseteq B(X))$ into $B(X)$. Then $\delta$ is quasispatial.

Proof. If $\operatorname{Imp}(\delta)$ is nonempty, then the lemma is trivially true. Therefore, for the rest of the argument, we assume that $\operatorname{Imp}(\delta)$ is empty.

First, we have that if $\delta$ is a transitive derivation from $\operatorname{Dom}(\delta)$ into $B(X)$, then

$$
\operatorname{Lat}\left(\mathcal{A}_{\delta}\right)=\{\{0\} \oplus\{0\}, X \oplus\{0\}, X \oplus X, G(T): T \in \operatorname{Imp}(\delta)\},
$$

where $G(T)=\left\{\left(\begin{array}{c}T(x) \\ x\end{array}\right): x \in \operatorname{Dom}(T)\right\}$ is the graph of $T$.

Indeed, it is easy to see that $\{0\} \oplus\{0\}, X \oplus\{0\}, X \oplus X$, and all $G(T)$ are invariant subspaces of $\mathcal{A}_{\delta}$. For the converse, suppose that $M \in$ Lat $\mathcal{A}_{\delta}$ such that $M \neq\{0\} \oplus\{0\}, M \neq X \oplus\{0\}$, and $M \neq X \oplus X$. If there is a vector of form $\left(\begin{array}{l}x \\ 0\end{array}\right) \in M$ with $x \neq 0$, then $\left(\begin{array}{c}A x \\ 0\end{array}\right) \in M$ for any $A \in \mathcal{A}$. It follows that $X \oplus\{0\} \subseteq M$ by the transitivity of $\operatorname{Dom}(\delta)$. If $M$ contains no vector of form $\left(\begin{array}{l}0 \\ x\end{array}\right)$ with $x \neq 0$, then $M=X \oplus\{0\}$; otherwise, there is a vector of form $\left(\begin{array}{l}0 \\ x\end{array}\right) \in M$ with $x \neq 0$, then we have that $M=X \oplus X$. Therefore $\left(\begin{array}{l}x \\ 0\end{array}\right) \in M$ implies that $x=0$. It follows that there is a closed operator $T$ such that

$$
M=G(T)=\left\{\left(\begin{array}{c}
T(x) \\
x
\end{array}\right): x \in \operatorname{Dom}(T)\right\}, \quad T \in \operatorname{Imp}(\delta) .
$$

As $\operatorname{Imp}(\delta)=\emptyset$, by (3.1),

$$
\begin{aligned}
\operatorname{Alg} \operatorname{Lat} \mathcal{A}_{\delta} & =\left\{\left(\begin{array}{ll}
A & B \\
0 & C
\end{array}\right): A, B, C \in B(X)\right\} \\
& \neq \mathcal{A}_{\delta}=\left\{\left(\begin{array}{cc}
A & \delta(A) \\
0 & A
\end{array}\right): A \in \operatorname{Dom}(\delta)\right\} .
\end{aligned}
$$

It is a contradiction to the assumption that $\delta$ is reflexive, which shows that

$$
\operatorname{Imp}(\delta) \neq \emptyset
$$

Therefore $\delta$ is quasispatial. 
Lemma 3.3. Let $\delta$ be a reflexive transitive derivation from $\operatorname{Dom}(\delta)$ into $B(X)$. Then $\delta=\delta_{\operatorname{Imp}(\delta)}$.

Proof. By (3.4), $\operatorname{Imp}(\delta) \neq \emptyset$. And by (2.4) of [5],

$$
\operatorname{Imp}(\delta)=\operatorname{Imp}\left(\delta_{\operatorname{Imp}(\delta)}\right)
$$

As both $\delta$ and $\delta_{\operatorname{Imp}(\delta)}$ are transitive derivations, Lat $\mathcal{A}_{\delta}=\operatorname{Lat} \mathcal{A}_{\delta_{\operatorname{Imp}(\delta)}}$ by (3.1). Since both of these derivations are reflexive,

$$
\mathscr{A}_{\delta}=\operatorname{Alg} \operatorname{Lat} \mathscr{A}_{\delta}=\operatorname{Alg} \operatorname{Lat} \mathscr{A}_{\delta_{\operatorname{Imp}(\delta)}}=\mathcal{A}_{\delta_{\operatorname{Imp}(\delta)}} .
$$

Hence

$$
\delta=\delta_{\operatorname{Imp}(\delta)}
$$

Lemma 3.4. Let $A$ be a transitive subalgebra of $B(X)$. If $\bar{A}$ contains a nonzero minimal left ideal $\supset$, then $\bar{A} \cap \mathcal{F}(X)$ is also a transitive subalgebra of $B(X)$.

Proof. We complete the proof step by step.

Step 1. We have that

$$
\supset^{2}=\left\{B_{1} B_{2}: B_{1}, B_{2} \in \mathcal{O}\right\} \neq\{0\} .
$$

Indeed, suppose that $\supset^{2}=\{0\}$. Let $0 \neq A_{0} \in \supset$. Then $\bar{A} A_{0} \subseteq$ O. It follows that $\left(\bar{A} A_{0}\right)^{2} \subseteq$ $\partial^{2}$ and $\left(\bar{A} A_{0}\right)^{2}=\{0\}$. Set $X_{0}=\{x \in X: A(x)=0$ for all $A \in \bar{A}\}$. It is obvious that $X_{0}$ is an $\bar{A}$ invariant closed subspace of $X$. Hence $X_{0}=\{0\}$ since $\mathscr{A}$ is a transitive operator algebra. Since $\left(\overline{\mathscr{A}} A_{0}\right)^{2}=\{0\}, A_{0} \overline{\mathscr{A}} A_{0}(X) \subseteq X_{0}$. It follows that $A_{0} \overline{\mathscr{A}} A_{0}(X)=\{0\}$ so that $A_{0} \overline{\mathscr{A}} A_{0}=\{0\}$. We can choose $x_{0} \in X\left(x_{0} \neq 0\right)$ such that $A_{0}\left(x_{0}\right) \neq 0$. It is obvious that $\bar{A} A_{0}\left(x_{0}\right)$ is an $\bar{A}$-invariant linear manifold of $X$ and $\bar{A} A_{0}\left(x_{0}\right) \neq\{0\}$. It follows that $\bar{A} A_{0}\left(x_{0}\right)$ is dense in $X$. However, $\bar{A} A_{0}\left(x_{0}\right)$ is contained in the null space of $A_{0}, A_{0}=0$, which is a contradiction.

Step 2. Since $\supset^{2} \neq\{0\}$, it follows from Lemma 2.1.5 and Corollary 2.1.6 of [16] that there exists an idempotent $P$ in $\bar{A}$ such that $\supset=\bar{A} P$ and $P \bar{A} P$ is a division algebra consisting of scalar multiples of $P$ (with identity $P$ ), that is,

$$
P \bar{A} P=\{\mu P: \mu \text { is a complex scalar }\}
$$

Then $P$ is a rank-one operator; that is, there exist $x_{1} \in X$ and $f \in X^{*}$ such that

$$
P=x_{1} \otimes f \in \overline{\mathcal{A}}, \quad f\left(x_{1}\right)=1 .
$$


Indeed, let $x_{1}: 0 \neq x_{1} \in X$ such that $P x_{1}=x_{1}$ and let $M_{1}=\bar{A}\left(x_{1}\right)=\left\{A\left(x_{1}\right): A \in \bar{A}\right\}$. It is obvious that $M_{1} \neq\{0\}$ and $M_{1}$ is an $\bar{A}$-invariant linear manifold of $X$. Hence $M_{1}$ is dense in X. Since $P A\left(x_{1}\right)=P A P\left(x_{1}\right)=\mu P\left(x_{1}\right)=\mu x_{1}$ for any $A \in \bar{A}$, the restriction $\left.P\right|_{M_{1}}$ of $P$ on $M_{1}$ has one-dimensional range. As $P$ is bounded, it also has one-dimensional range. Hence $P=x_{1} \otimes f$, where $f$ is a continuous functional.

Step 3. We have $P=x_{1} \otimes f \in \supset \subseteq \overline{\mathcal{A}}$. As $\bar{A}$ is an algebra, the set of rank-one operators

$$
\mathcal{S}_{P}=\{A P B: A, B \in \mathcal{A}\}=\left\{A x_{1} \otimes B^{*} f: A, B \in \mathcal{A}\right\}
$$

lies in $\overline{\mathscr{A}} \cap \mathcal{F}(X)$. For each $0 \neq y \in X$, the linear manifold

$$
M_{y}=\left\{\sum_{i=1}^{n} S_{i} y: S_{i} \in S_{P}, i=1,2, \ldots, n, n=1,2, \ldots\right\}
$$

is dense in $X$. Indeed, if $M_{y}$ is not dense in $X$, by Hahn-Banach theorem, there exists $0 \neq g \in$ $X^{*}$ such that $g(z)=0$ for all $z \in M_{y}$. Hence

$$
0=g\left(\left(A x_{1} \otimes B^{*} f\right) y\right)=g\left(\left(B^{*} f(y)\right) A x_{1}\right)=f(B y) g\left(A x_{1}\right) \quad \text { for any } A, B \in \mathcal{A} \text {. }
$$

If $f(B y)=0$ for all $B \in \mathcal{A}$, then $f$ vanishes on the linear manifold $\mathcal{A} y=\{B y: B \in \mathcal{A}\}$. However, as $\mathcal{A}$ is transitive, the manifold $\mathcal{A} y$ is dense in $X$, so that $f=0$. This contradiction shows that there is $B_{1} \in \mathcal{A}$ such that $f\left(B_{1} y\right) \neq 0$. Then (3.13) implies that $g\left(A x_{1}\right)=0$ for all $A \in \mathcal{A}$. Repeating the above argument, we obtain that $g=0$. This contradiction shows that any manifold $M_{y}$ is dense in X. Therefore the algebra $\bar{A} \cap \mathcal{F}(X)$ is transitive.

Lemma 3.5. Suppose that $\mathbb{A}$ is a transitive subalgebra of $B(X)$ and $\bar{A}$ contains a nonzero minimal left ideal $\supset$. Then the operators commuting with $\bar{A}$ are scalars. More precisely, if $T$ is a closed, densely defined operator such that $A(\operatorname{Dom}(T)) \subseteq \operatorname{Dom}(T)$ and $A T \xi=T A \xi$ for any $A \in \bar{A}$ and any $\xi \in$ $\operatorname{Dom}(T)$, then $T=\mu I$ for some complex scalar $\mu$.

Proof. For any $y \in X$, there exists a net of operators $\left\{A_{\lambda}\right\} \subseteq \mathcal{A}$ such that $\lim _{\lambda} A_{\lambda}\left(x_{1}\right)=y$ by the transitivity of $\mathscr{A}$. Then $\overline{\mathscr{A}} \ni A_{\curlywedge}\left(x_{1} \otimes f\right)=A_{\curlywedge}\left(x_{1}\right) \otimes f \stackrel{\|\cdot\|}{\longrightarrow} y \otimes f$, since $P=x_{1} \otimes f \in \bar{A}$ as in (3.10). It follows that

$$
y \otimes f \in \bar{A} \text { for any } y \in X
$$

Suppose that $T$ is a closed, densely defined operator commuting with $\bar{A}$. As $S=(y \otimes$ $f) \in \bar{A}$ for any $y \in X$, then $(y \otimes f)(\xi) \in \operatorname{Dom}(T)$ and $(y \otimes f) T \xi=T(y \otimes f) \xi$ for any $\xi \in \operatorname{Dom}(T)$. Since $\operatorname{Dom}(T)$ is dense in $X$ and $f \neq 0$, there is $\xi_{0} \in \operatorname{Dom}(T)$ such that $f\left(\xi_{0}\right) \neq 0$. So

$$
(y \otimes f)\left(\xi_{0}\right)=f\left(\xi_{0}\right) y \in \operatorname{Dom}(T), \quad f\left(T \xi_{0}\right) y=f\left(\xi_{0}\right) T(y), \quad \text { for any } y \in X
$$

It follows that $\operatorname{Dom}(T)=X$. Set $\mu=f\left(T \xi_{0}\right) / f\left(\xi_{0}\right)$. Then $T=\mu I$, as required. 
Lemma 3.6. Suppose that $\mathcal{A}$ is a subalgebra of $B(X)$ and $\bar{A}$ is its norm closure. Then Lat $(\mathcal{A})=$ $\operatorname{Lat}(\bar{A})$.

Proof. Clearly, $\operatorname{Lat}(\overline{\mathcal{A}}) \subseteq \operatorname{Lat}(\mathcal{A})$. Conversely, let $M \in \operatorname{Lat}(\mathcal{A})$. For any $B \in \overline{\mathcal{A}}$, there exists a net of operators $\left\{A_{\curlywedge}\right\} \subseteq \mathcal{A}$ such that $A_{\curlywedge} \stackrel{\|\cdot\|}{\longrightarrow} B$. Then $A_{\curlywedge} x \in M$ for any $x \in M$. Since $A_{\curlywedge} x \rightarrow B x$ and $M$ is a closed subspace of $X, B x \in M$. It follows that $M \in \operatorname{Lat}(B)$ so that $M \in \operatorname{Lat}(\bar{A})$. Therefore $\operatorname{Lat}(\mathcal{A}) \subseteq \operatorname{Lat}(\overline{\mathcal{A}})$. The Lemma follows.

Let $\mathcal{A}$ be a subalgebra of $B(X)$ and $\delta$ be a bounded derivation from $\mathcal{A}(\mathcal{A}=\operatorname{Dom}(\delta))$ into $B(X)$. For any $B \in \bar{A}$, there exists a net of operators $\left\{A_{\curlywedge}\right\} \subseteq \mathcal{A}$ such that $A_{\curlywedge} \stackrel{\|\cdot\|}{\longrightarrow} B$. If $\left\{A_{\curlywedge}\right\}$ is a uniformly convergent net in $A$; then $\left\{\delta\left(A_{\curlywedge}\right)\right\}$ is also a uniformly convergent net in $B(X)$ since $\left\|\delta\left(A_{\lambda_{1}}\right)-\delta\left(A_{\lambda_{2}}\right)\right\| \leq\|\delta\|\left\|A_{\lambda_{1}}-A_{\lambda_{2}}\right\|$ for any indexes $\lambda_{1}, \lambda_{2}$. Also, if two nets of operators $\left\{A_{\lambda}^{(1)}\right\},\left\{A_{\lambda}^{(2)}\right\}$ in $\mathcal{A}$ converge uniformly to the same limit, then $\left\{\delta\left(A_{\lambda}^{(1)}\right)\right\},\left\{\delta\left(A_{\lambda}^{(2)}\right)\right\}$ converge uniformly to the same limit, since $\left\|\delta\left(A_{\lambda}^{(1)}\right)-\delta\left(A_{\lambda}^{(2)}\right)\right\| \leq\|\delta\|\left\|A_{\lambda}^{(1)}-A_{\lambda}^{(2)}\right\|$. Therefore a linear map $\bar{\delta}$ can be unambiguously defined by

$$
\operatorname{Dom}(\bar{\delta})=\overline{\operatorname{Dom}(\delta)}=\bar{A}, \quad \bar{\delta}(B)=\lim _{\lambda} \delta\left(A_{\curlywedge}\right)
$$

for any $B \in \bar{A}$ and any net $\left\{A_{\curlywedge}\right\}$ in $\mathcal{A}$ such that $A_{\curlywedge} \stackrel{\|\cdot\|}{\longrightarrow} B$, where $\bar{\delta}(B)$ is the limit of $\delta\left(A_{\curlywedge}\right)$ in the operator norm topology. It is obvious that $\|\bar{\delta}\|=\|\delta\|$ if $\delta$ is bounded.

Proposition 3.7. Let $\delta$ be a bounded transitive derivation from $\mathcal{A}(\mathcal{A}=\operatorname{Dom}(\delta))$ into $B(X)$. Then $\bar{\delta}$ is a bounded transitive derivation from $\bar{A}$ into $B(X)$ and

$$
\operatorname{Imp}(\bar{\delta})=\operatorname{Imp}(\delta)
$$

Proof. Since $A$ is transitive, $\bar{A}$ is also transitive. It is obvious that $\bar{\delta}$ is a linear map. For any $B_{1}, B_{2} \in \bar{A}$, there are two nets of operators $\left\{A_{\lambda}^{(1)}\right\},\left\{A_{\lambda}^{(2)}\right\}$ in $\mathcal{A}$ such that $A_{\lambda}^{(i)} \stackrel{\|\cdot\|}{\longrightarrow} B_{i}(i=1,2)$. Then $A_{\lambda}^{(1)} A_{\lambda}^{(2)} \stackrel{\|\cdot\|}{\longrightarrow} B_{1} B_{2}$ and

$$
\bar{\delta}\left(B_{1} B_{2}\right)=\lim _{\lambda} \delta\left(A_{\lambda}^{(1)} A_{\lambda}^{(2)}\right)=\lim _{\lambda} \delta\left(A_{\lambda}^{(1)}\right) A_{\lambda}^{(2)}+\lim _{\lambda} A_{\lambda}^{(1)} \delta\left(A_{\lambda}^{(2)}\right)=\bar{\delta}\left(B_{1}\right) B_{2}+B_{1} \bar{\delta}\left(B_{2}\right) .
$$

It follows that the linear map $\bar{\delta}$ is a transitive derivation from $\bar{A}$ into $B(X)$.

By (2.2), $\mathcal{A}_{\delta}=\left\{\left(\begin{array}{cc}A & \delta(A) \\ 0 & A\end{array}\right): A \in \mathcal{A}\right\}$ and $\mathcal{A}_{\bar{\delta}}=\left\{\left(\begin{array}{cc}B \bar{\delta}(B) \\ 0 & B\end{array}\right): B \in \overline{\mathcal{A}}\right\}$. So that $\mathcal{A}_{\bar{\delta}}=\overline{A_{\delta}}$ by (3.16) and $\operatorname{Lat}\left(\mathcal{A}_{\bar{\delta}}\right)=\operatorname{Lat}\left(\mathcal{A}_{\delta}\right)$ by Lemma 3.6. By (3.1),

$$
\begin{gathered}
\operatorname{Lat}\left(\mathcal{A}_{\delta}\right)=\{\{0\} \oplus\{0\}, X \oplus\{0\}, X \oplus X, G(F): F \in \operatorname{Imp}(\delta)\}, \\
\operatorname{Lat}\left(\mathcal{A}_{\bar{\delta}}\right)=\left\{\{0\} \oplus\{0\}, X \oplus\{0\}, X \oplus X, G\left(F_{1}\right): F_{1} \in \operatorname{Imp}(\bar{\delta})\right\} .
\end{gathered}
$$

It follows that $\operatorname{Imp}(\bar{\delta})=\operatorname{Imp}(\delta)$. 
Proof of Theorem 3.1. By (3.4), $\operatorname{Imp}(\delta) \neq \emptyset$. Suppose that $T \in \operatorname{Imp}(\delta)$. Then $T$ is a closed, densely defined operator on $X$ and

$$
\{(T+\mu I): \mu \text { is a complex scalar }\} \subseteq \operatorname{Imp}(\delta) .
$$

Let $\delta_{0}=0$ be the derivation (with the same domain $\mathcal{A}$ of $\delta$ ) from $\mathcal{A}$ into $B(X)$, that is, $\delta_{0}(A)=0$ for any $A \in \mathcal{A}$. If $T_{0} \in \operatorname{Imp}\left(\delta_{0}\right)$ with domain $\operatorname{Dom}\left(T_{0}\right)$, then $T_{0} \in \operatorname{Imp}\left(\overline{\delta_{0}}\right)$ by (3.17), and

$$
A\left(\operatorname{Dom}\left(T_{0}\right)\right) \subseteq \operatorname{Dom}\left(T_{0}\right), \quad \overline{\delta_{0}}(A) x=\left(T_{0} A-A T_{0}\right) x \quad \text { for any } A \in \overline{\mathcal{A}}, x \in \operatorname{Dom}\left(T_{0}\right),
$$

that is, $T_{0} A x=A T_{0} x$ for any $x \in \operatorname{Dom}\left(T_{0}\right)$. Then $T_{0}=\mu I$ for some scalar $\mu$ by Lemma 3.5. It follows that

$$
\operatorname{Imp}\left(\delta_{0}\right)=\{\mu I: \mu \text { is a complex scalar }\}
$$

Let $T_{1} \in \operatorname{Imp}(\delta)$ be any closed, densely defined operators implementing $\delta$. Then $T_{1} \in$ $\operatorname{Imp}(\bar{\delta})$ by (3.17). Then $\operatorname{Dom}\left(T_{1}\right)$ is a nonzero $\operatorname{Dom}(\bar{\delta})$-invariant linear manifold of $X$ by (1.3); that is, $\operatorname{Dom}\left(T_{1}\right)$ is a nonzero $\bar{A}$-invariant linear manifold of $X$. Therefore $A\left(\operatorname{Dom}\left(T_{1}\right)\right) \subseteq$ $\operatorname{Dom}\left(T_{1}\right)$ for any $A \in \overline{\mathcal{A}}$. If $y \in X, y \otimes f \in \bar{A}$ by (3.14) and $(y \otimes f) \xi=f(\xi) y \in \operatorname{Dom}\left(T_{1}\right)$ for any $\xi \in \operatorname{Dom}\left(T_{1}\right)$. Since $\operatorname{Dom}\left(T_{1}\right)$ is dense in $X$ and $f \neq 0$, there is $\xi_{0} \in \operatorname{Dom}\left(T_{1}\right)$ such that $f\left(\xi_{0}\right) \neq 0$. So $y \in \operatorname{Dom}\left(T_{1}\right)$. Since $y$ is arbitrary chosen, it follows that $\operatorname{Dom}\left(T_{1}\right)=X$, the whole space. Then $\operatorname{Dom}(T)=X$ also holds since $T_{1}$ is an arbitrary implementation of $\delta$. It is obvious that $\operatorname{Dom}\left(T_{1}-T\right)=\left(\operatorname{Dom}(T) \cap \operatorname{Dom}\left(T_{1}\right)\right)=X$. Therefore the operator $T_{1}-T$ is everywhere defined.

Set $T_{2}=T_{1}-T$. Then $T_{2}$ is closable.

Indeed, suppose that $\xi_{n} \in X, \xi_{n} \rightarrow 0$ and $T_{2}\left(\xi_{n}\right) \rightarrow \eta$. Since $T, T_{1} \in \operatorname{Imp}(\bar{\delta}), \bar{\delta}(A) x=$ $\left(T_{1} A-A T_{1}\right) x$ and $\bar{\delta}(A) x=(T A-A T) x$ for any $A \in \operatorname{Dom}(\bar{\delta})$ and any $x \in X$. So $T_{2} A \xi_{n}=A T_{2} \xi_{n}$ for any $A \in \bar{A}$. As $P=x_{1} \otimes f \in \bar{A}$ by (3.10) and $\bar{A}$ is an algebra, $T_{2} P A \xi_{n}=P A T_{2} \xi_{n}$ for any $A \in$ $\bar{A}$. Then

$$
\begin{aligned}
f(A \eta) x_{1} & =\left(x_{1} \otimes f\right) A \eta=P A \eta=\lim _{n \rightarrow \infty} P A T_{2} \xi_{n}=\lim _{n \rightarrow \infty} T_{2} P A \xi_{n} \\
& =\lim _{n \rightarrow \infty} T_{2}\left(x_{1} \otimes f\right) A \xi_{n}=\lim _{n \rightarrow \infty} f\left(A \xi_{n}\right) T_{2} x_{1}=0
\end{aligned}
$$

for any $A \in \bar{A}$. Since $x_{1} \neq 0, f(A \eta)=0$ for any $A \in \bar{A}$. Thus $f$ vanishes on the linear manifold $\bar{A} \eta=\{A \eta: A \in \bar{A}\}$. The transitivity of $\bar{A}$ assures that $\bar{A} \eta=\{0\}$ or $\bar{A} \eta$ is dense in X. Since $f \neq 0, \bar{A} \eta=\{0\}$ and $\eta=0$.

It follows that $T_{2}$ is a closable operator with domain $\operatorname{Dom}\left(T_{2}\right)=X$, the whole space. So that $T_{2}$ is a closed operator. Clearly, $T_{2}=T_{1}-T \in \operatorname{Imp}\left(\delta_{0}\right)$. Then $T_{1}-T=\mu I$ for some scalar $\mu$ by (3.22), that is, $T_{1}=T+\mu I$. It follows that

$$
\operatorname{Imp}(\delta)=\{(T+\mu I): \mu \text { is a complex scalar }\}
$$


Since $\Delta_{T}=\Delta_{T+\mu I}$, by (3.7),

$$
\delta=\delta_{\operatorname{Imp}(\delta)}=\Delta_{T}
$$

Since $T$ is closed and $\operatorname{Dom}(T)=X, T$ is bounded by the closed graph theorem. It follows from (2.4), (3.24), and (3.25) that $\delta(A)=T A-A T$ for each $A \in \mathcal{A}$ and the implementation $T$ is unique only up to an additive constant.

\section{Applications}

Suppose that $\mathcal{A}$ is a subalgebra of $B(X)$ and $\delta$ is a derivation from $\mathcal{A}(\mathcal{A}=\operatorname{Dom}(\delta))$ into $B(X)$. We can define the adjoint $\delta^{*}$ of $\delta$ by

$$
\operatorname{Dom}\left(\delta^{*}\right)=(\mathcal{A})^{*}=\left\{A^{*}: A \in \mathcal{A}\right\}, \quad \delta^{*}\left(A^{*}\right)=(\delta(A))^{*} \quad \text { for any } A \in \mathcal{A} .
$$

Lemma 4.1. Let $\delta$ be a derivation from $\mathcal{A}(\mathcal{A}=\operatorname{Dom}(\delta))$ into $B(X)$.

(1) The adjoint $\delta^{*}$ of $\delta$ is a derivation from $\mathcal{A}^{*}$ into $B\left(X^{*}\right)$.

(2) Furthermore, suppose that $X$ is a reflexive Banach space and $\mathcal{A}$ is a subalgebra of $B(X)$. Then $\delta^{*}$ is a reflexive (resp., transitive) derivation from $\mathcal{A}^{*}$ into $B\left(X^{*}\right)$ if and only if $\delta$ is a reflexive (resp., transitive) derivation from $\mathcal{A}$ into $B(X)$. And $\operatorname{Imp}\left(\delta^{*}\right)=\left\{-T^{*}: T \in\right.$ $\operatorname{Imp}(\delta)\}$.

Proof. (1) It is obvious that $\delta^{*}$ is a linear map from $A^{*}$ into $B\left(X^{*}\right)$. If $A_{1}^{*}, A_{2}^{*} \in \operatorname{Dom}\left(\delta^{*}\right)$, then

$$
\begin{aligned}
\delta^{*}\left(A_{1}^{*} A_{2}^{*}\right) & =\delta^{*}\left(\left(A_{2} A_{1}\right)^{*}\right)=\left(\delta\left(A_{2} A_{1}\right)\right)^{*}=\left(\delta\left(A_{2}\right) A_{1}+A_{2} \delta\left(A_{1}\right)\right)^{*} \\
& =\mathrm{A}_{1}^{*}\left(\delta\left(A_{2}\right)\right)^{*}+\left(\delta\left(A_{1}\right)\right)^{*} A_{2}^{*}=A_{1}^{*}\left(\delta^{*}\left(A_{2}^{*}\right)\right)+\left(\delta^{*}\left(A_{1}^{*}\right)\right) A_{2}^{*}
\end{aligned}
$$

It follows that $\delta^{*}$ is a derivation.

(2) Suppose that $\delta$ is transitive. Then $\mathscr{A}$ is transitive and Lat $\mathcal{A}=\{X,\{0\}\}$. Since $M \in$ Lat $A$ if and only if $M^{\perp} \in \operatorname{Lat}\left(A^{*}\right)$ for any $A \in B(X)$,

$$
\operatorname{Lat}\left(\operatorname{Dom}\left(\delta^{*}\right)\right)=\operatorname{Lat}\left(\mathscr{A}^{*}\right)=\left\{M^{\perp}: M \in \operatorname{Lat} \mathscr{A}\right\}=\left\{\{0\}^{\perp}, X^{\perp}\right\}=\left\{X^{*},\{0\}\right\}
$$

We have that $\operatorname{Dom}\left(\delta^{*}\right)$ is transitive, that is, $\delta^{*}$ is transitive.

Conversely, suppose that $\delta^{*}$ is transitive. We obtain, as above, that $\delta^{* *}$ is transitive. Since $\mathrm{X}$ is a reflexive Banach space, $A^{* *}=A$ for any bounded operator $A \in B(X)$. It follows from (4.1) that $\delta=\delta^{* *}$ is transitive by the definition of $\delta^{*}$. 

Since

Set $J=\left(\begin{array}{ll}0 & I \\ I & 0\end{array}\right)$, where $I$ is the identity operator on $X^{*}$ and 0 is the zero operator on $X^{*}$.

$$
\begin{aligned}
\mathscr{A}_{\delta^{*}} & =\left\{\left(\begin{array}{cc}
A^{*} & \delta^{*}\left(A^{*}\right) \\
0 & A^{*}
\end{array}\right): A \in \mathcal{A}\right\}=\left\{J\left(\begin{array}{cc}
A^{*} & 0 \\
\delta(A)^{*} & A^{*}
\end{array}\right) J: A \in \mathcal{A}\right\} \\
& =\left\{J\left(\begin{array}{cc}
A & \delta(A) \\
0 & A
\end{array}\right) J: A \in \mathcal{A}\right\}=J\left(\mathcal{A}_{\delta}\right)^{*} J=J^{-1}\left(\mathcal{A}_{\delta}\right)^{*} J,
\end{aligned}
$$

the two operator algebras $\mathcal{A}_{\delta^{*}}$ and $\left(\mathcal{A}_{\delta}\right)^{*}$ are similar. We have that

$$
\operatorname{Lat}\left(\mathcal{A}_{\delta^{*}}\right)=J\left(\operatorname{Lat}\left(\left(\mathscr{A}_{\delta}\right)^{*}\right)\right)=J\left(\operatorname{Lat}\left(\mathscr{A}_{\delta}\right)\right)^{\perp}
$$

It is obvious that

$$
\begin{array}{ll}
(X \oplus X)^{\perp}=\{0\} \oplus\{0\}, \quad(\{0\} \oplus\{0\})^{\perp}=X^{*} \oplus X^{*}, \\
(X \oplus\{0\})^{\perp}=\{0\} \oplus X^{*}, \quad J\left(\{0\} \oplus X^{*}\right)=X^{*} \oplus\{0\} .
\end{array}
$$

If $T \in \operatorname{Imp}(\delta),\left(\begin{array}{c}g \\ -T^{*}(g)\end{array}\right) \in(G(T))^{\perp}$ for any $g \in \operatorname{Dom}\left(T^{*}\right)$, since

$$
G(T)=\left\{\left(\begin{array}{c}
T(x) \\
x
\end{array}\right): x \in \operatorname{Dom}(T)\right\}, \quad g(T(x))+\left(-T^{*}(g)\right)(x)=0 \quad \text { for any } x \in \operatorname{Dom}(T) \text {. }
$$

Conversely, if $g, h \in X^{*}$ such that $\left(\begin{array}{l}g \\ h\end{array}\right) \in(G(T))^{\perp}$, then

$$
g(T x)+h(x)=0 \quad \text { for any } x \in \operatorname{Dom}(T)
$$

So that the functional $x \mapsto g(T x)(=-h(x))$ is a bounded functional on $\operatorname{Dom}(T)$. Therefore $g \in \operatorname{Dom}\left(T^{*}\right)$ and $h=\left(-T^{*}(g)\right)$ holds on $\operatorname{Dom}(T)$ by $(4.8)$. As $\left(-T^{*}(g)\right)$ and $h$ are bounded functionals on $X$ and $\operatorname{Dom}(T)$ is dense in $X, h=\left(-T^{*}(g)\right)$ on $X$. It follows that

$$
\begin{gathered}
(G(T))^{\perp}=\left\{\left(\begin{array}{c}
g \\
-T^{*}(g)
\end{array}\right): g \in \operatorname{Dom}\left(T^{*}\right)\right\}, \\
J(G(T))^{\perp}=\left\{\left(\begin{array}{c}
-T^{*}(g) \\
g
\end{array}\right): g \in \operatorname{Dom}\left(T^{*}\right)\right\}=G\left(-T^{*}\right) .
\end{gathered}
$$

Hence

$$
\operatorname{Lat}\left(\mathscr{A}_{\delta^{*}}\right)=\left\{\{0\} \oplus\{0\}, X^{*} \oplus\{0\}, X^{*} \oplus X^{*}, G\left(-T^{*}\right): T \in \operatorname{Imp}(\delta)\right\}
$$


It follows from (3.4) that

$$
\operatorname{Imp}\left(\delta^{*}\right)=\left\{-T^{*}: T \in \operatorname{Imp}(\delta)\right\}
$$

Suppose that $\delta$ is reflexive. Then $A_{\delta}$ is a reflexive operator algebra on $X \oplus X$. Since $\mathscr{A}_{\delta^{*}}=J^{-1}\left(\mathscr{A}_{\delta}\right)^{*} J$, the two operator algebras $\mathscr{A}_{\delta^{*}}$ and $\left(\mathscr{A}_{\delta}\right)^{*}$ are similar. It follows that $\mathscr{A}_{\delta^{*}}$ is reflexive if and only if $\left(\mathscr{A}_{\delta}\right)^{*}$ is reflexive. Since $\mathscr{A}_{\delta}$ is reflexive, $\left(\mathscr{A}_{\delta}\right)^{*}$ is also reflexive by the reflexivity of the Banach space $X \oplus X$. Thus $\mathcal{A}_{\delta^{*}}$ is reflexive, that is, $\delta^{*}$ is reflexive.

Conversely, suppose that $\delta^{*}$ is reflexive. We obtain, as above, that $\delta^{* *}$ is reflexive. It follows that $\delta=\delta^{* *}$ is reflexive.

Theorem 4.2. Suppose that $X$ is a reflexive Banach space. Let $A$ be a transitive subalgebra of $B(X)$ and let $\bar{A}$ contain a nonzero minimal right ideal 0 . If $\delta$ is a bounded reflexive transitive derivation from $A$ into $B(X)$, then $\delta$ is spatial and implemented uniquely, or more precisely, there exists $T \in B(X)$ such that $\delta(A)=T A-A T$ for each $A \in \mathcal{A}$, and the implementation $T$ is unique only up to an additive constant.

Proof. Consider the derivation $\delta^{*} . \delta^{*}$ is a bounded reflexive transitive derivation from $\mathscr{A}^{*}=$ $\left\{A^{*}: A \in \mathcal{A}\right\}$ into $B\left(X^{*}\right)$ by Lemma 4.1.

Set $\supset^{*}=\left\{B^{*}: B \in \supset\right\}$. Since $\supset$ is a right ideal of $\bar{A}, B A \in \supset$ for any $B \in \supset$ and $A \in \bar{A}$. For

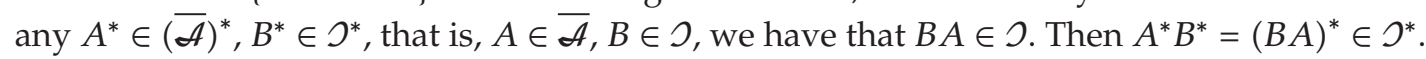
It follows that $\supset^{*}$ is a left ideal of $(\bar{A})^{*}$. If there exists a nonzero left ideal $\supset_{1}$ of $(\bar{A})^{*}$ included in $\supset^{*}$, then $\supset_{1}^{*}$ is a nonzero right ideal of $\bar{A}$ included in $\supset$. Thus $\supset_{1}^{*}=\supset$ and $\supset_{1}=\supset^{*}$. It follows that $\supset^{*}$ is a minimal left ideal of $(\overline{\mathscr{A}})^{*}=\overline{\mathcal{A}^{*}}$.

By (3.24), there exists a bounded operator $T_{1} \in B\left(X^{*}\right)$ such that

$$
\operatorname{Imp}\left(\delta^{*}\right)=\left\{\left(T_{1}+\mu I\right): \mu \text { is a complex scalar }\right\},
$$

where $I$ is the identity operator on $X^{*}$. By (4.11),

$$
\operatorname{Imp}(\delta)=\operatorname{Imp}\left(\delta^{* *}\right)=\left\{\left(-T_{1}^{*}-\bar{\mu} I\right): \mu \text { is a complex scalar }\right\},
$$

where $I$ is the identity operator on $X$. Set $T=-T_{1}^{*}$. Then $T$ is bounded with $\operatorname{Dom}(T)=X$. By (3.7) and (4.13), $\delta=\delta_{\operatorname{Imp}(\delta)}=\Delta_{T}$. It follows from (2.4) that $\delta(A)=T A-A T$ for each $A \in \mathcal{A}$, and the implementation $T$ is unique only up to an additive constant.

Corollary 4.3 (Proposition 2.11, [5]). Suppose that $\mathcal{A}$ is a transitive subalgebra of $B(H)$. Let $\delta$ be a bounded reflexive transitive derivation from $\mathcal{A}$ into $B(H)$. If $C(H) \subseteq \bar{A}$, then there exists $T \in B(H)$ such that $\delta(A)=T A-A T$ for each $A \in \mathcal{A}$, and the implementation $T$ is unique only up to an additive constant.

Proof. Since $C(H) \subseteq \bar{A}$, for a fixed vector $f \in H$,

$$
\supset=\{x \otimes f \mid x \in H\}
$$


is a minimal left ideal of $\overline{\mathcal{A}}$, where $x \otimes f$ is the rank-one operator on $H$ with $(x \otimes f) h=(h, f) x$ for $h \in H$. By Theorem 3.1, there exists $T \in B(H)$ such that $\delta(A)=T A-A T$ for each $A \in \mathcal{A}$, and the implementation $T$ is unique only up to an additive constant.

Remark 4.4. In a Banach space without the approximation property (there exists such space as this, e.g., [17]), not all compact operators can be approximated by finite-rank operators in the norm operator topology. Therefore, Theorems 3.1 and 4.2 of this paper improve [5, Proposition 2.11].

\section{Acknowledgments}

This paper was supported by the National Nature Science Foundation of China (Grant no. 11071188). The first author would like to express deep gratitude to Professor Kissin for helpful answer to the questions run across during the work. The authors are greatly indebted to the referees for carefully reading the paper and providing many helpful comments which lead to great improvement of the paper, and pointing out a major error in the original version of this paper.

\section{References}

[1] S. Albeverio, S. A. Ayupov, K. K. Kudaybergenov, and B. O. Nurjanov, "Local derivations on algebras of measurable operators," http:/ / arxiv.org/abs/0901.2983.

[2] S. Albeverio, S. A. Ayupov, and K. K. Kudaybergenov, "Structure of derivations on various algebras of measurable operators for type I von Neumann algebras," Journal of Functional Analysis, vol. 256, no. 9, pp. 2917-2943, 2009.

[3] E. Christensen, "Derivations of nest algebras," Mathematische Annalen, vol. 229, no. 2, pp. 155-161, 1977.

[4] P. R. Chernoff, "Representations, automorphisms, and derivations of some operator algebras," Journal of Functional Analysis, vol. 12, pp. 275-289, 1973.

[5] E. Kissin, "Reflexive *-derivations and lattices of invariant subspaces of operator algebras associated with them," Journal of Functional Analysis, vol. 232, no. 1, pp. 56-89, 2006.

[6] F. Lu, "Derivations of CDC algebras," Journal of Mathematical Analysis and Applications, vol. 323, no. 1, pp. 179-189, 2006.

[7] P. Li and J. Ma, "Derivations, local derivations and atomic Boolean subspace lattices," Bulletin of the Australian Mathematical Society, vol. 66, no. 3, pp. 477-486, 2002.

[8] P. Li, J. Ma, and J. Wu, "Additive derivations of certain reflexive algebras," Houston Journal of Mathematics, vol. 32, no. 2, pp. 521-530, 2006.

[9] D. Larson and A. Sourour, "Local derivations and local automorphisms of $B(X)$," Proceedings of Symposia in Pure Mathematics, vol. 51, pp. 187-194, 1990.

[10] R. L. Moore, “Derivations of CSL algebras," Indiana University Mathematics Journal, vol. 54, no. 6, pp. 1739-1750, 2005.

[11] M. Spivack, "Certain properties of derivations," Proceedings of the American Mathematical Society, vol. 99, no. 4, pp. 712-718, 1987.

[12] S. Sakai, $C^{*}$-Algebra and $W^{*}$-Algebra, Springer, Berlin, Germany, 1971.

[13] S. Sakai, "Derivations of $W^{*}$-algebras," Annals of Mathematics, vol. 83, pp. 273-279, 1966.

[14] K. R. Davidson, Nest Algebras, vol. 191 of Pitman Research Notes in Mathematics Series, Longman Scientific \& Technical, Harlow, UK, 1988.

[15] W. Rudin, Functional Analysis, McGraw-Hill, New York, NY, USA, 2nd edition, 1991.

[16] C. E. Rickart, Banach Algebras, The University Series in Higher Mathematics, D. van Nostrand Co., Princeton, NJ, USA, 1960.

[17] P. Enflo, "A counterexample to the approximation problem in Banach spaces," Acta Mathematica, vol. 130, pp. 309-317, 1973. 


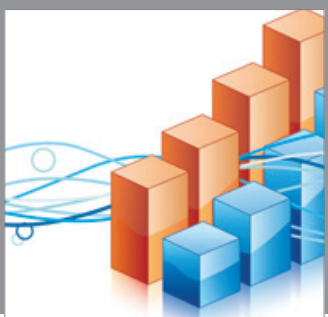

Advances in

Operations Research

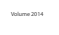

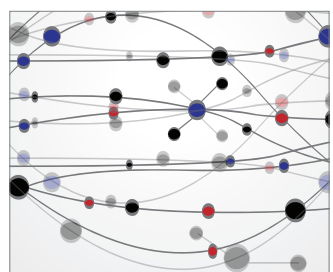

\section{The Scientific} World Journal
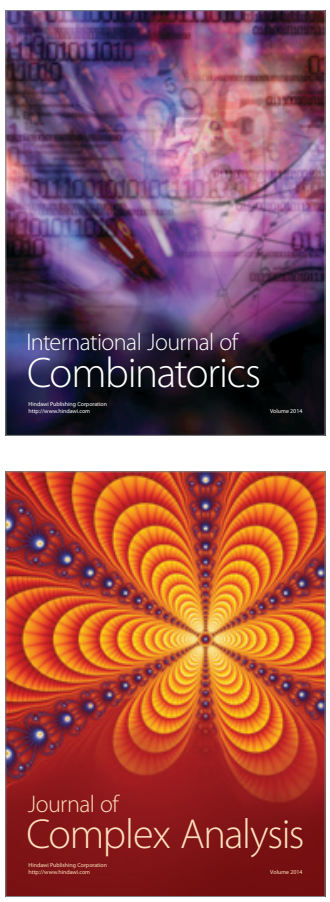

International Journal of

Mathematics and

Mathematical

Sciences
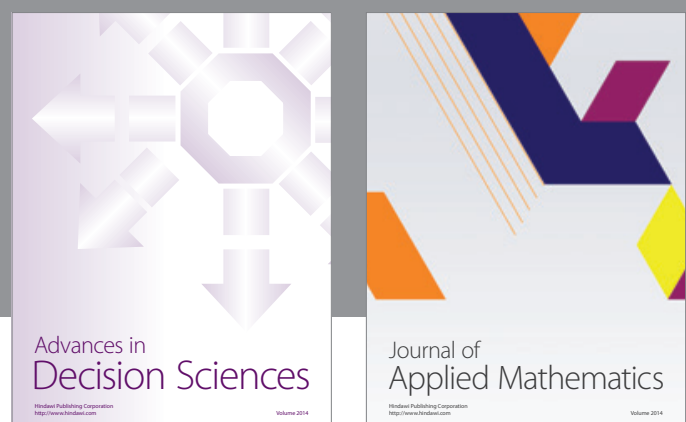

Journal of

Applied Mathematics
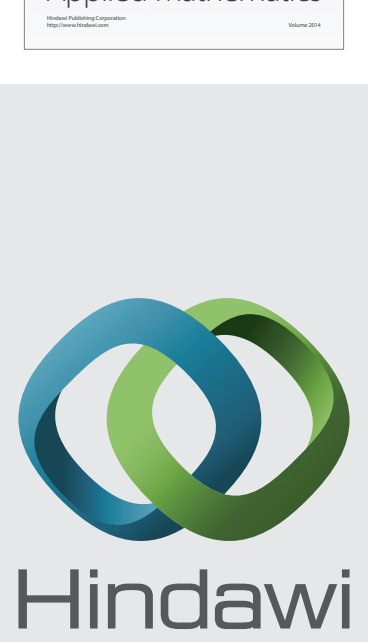

Submit your manuscripts at http://www.hindawi.com
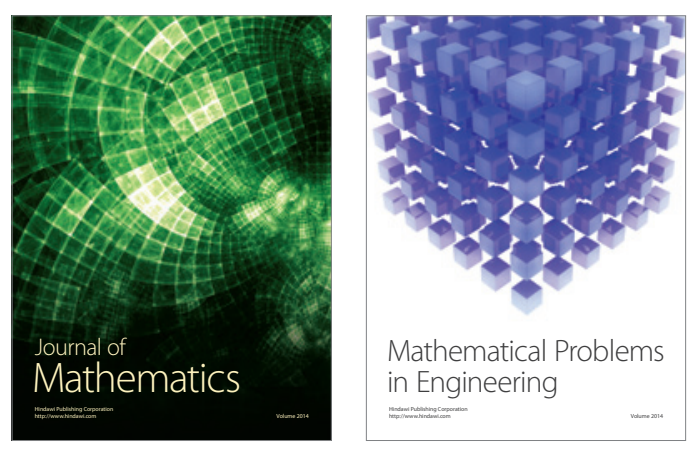

Mathematical Problems in Engineering
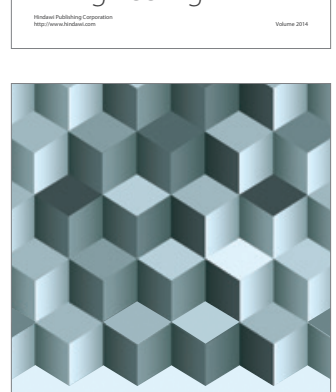

Journal of

Function Spaces
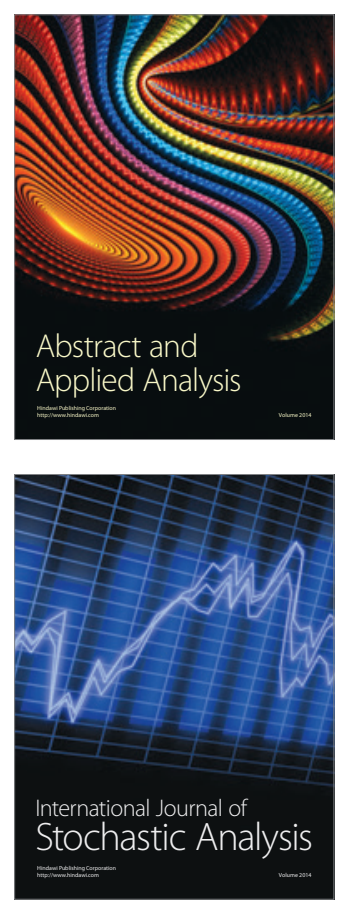

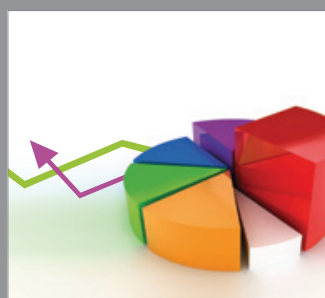

ournal of

Probability and Statistics

Promensencen
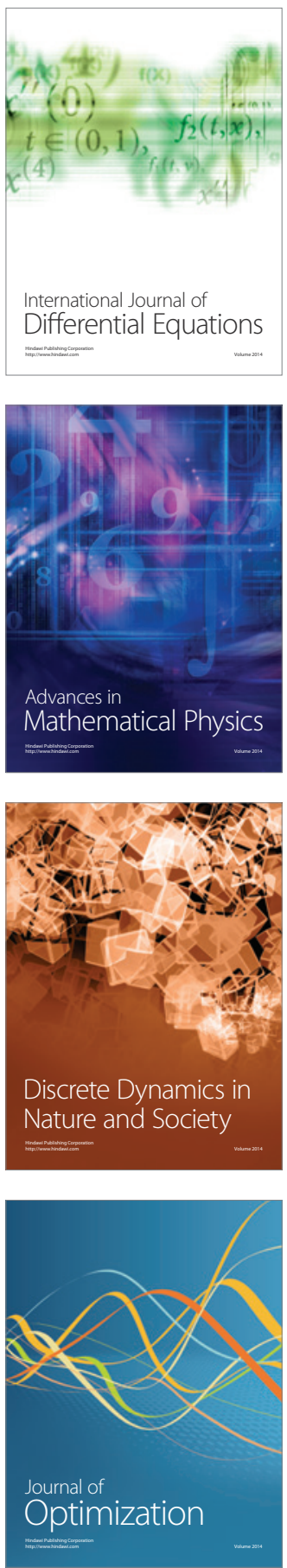